\title{
Quality of Local Treatment or Biology of the Tumor: Which are the Trump Cards for Loco-regional Control of Retroperitoneal Sarcoma?
}

\author{
Alessandro Gronchi, $\mathrm{MD}^{1}$ and Raphael E. Pollock, MD, $\mathbf{P h D}^{2}$ \\ ${ }^{1}$ Sarcoma Service, Department of Surgery, Istituto Nazionale Tumori, Milan, Italy; ${ }^{2}$ Department of Surgical Oncology, \\ University of Texas MD Anderson Cancer Center, Houston, TX
}

Surgery remains the mainstay of curative therapy for retroperitoneal sarcoma (RPS). Local control is critical to achieve this objective, yet is not possible to achieve even initially in as many as $50 \%$ of patients. Unlike sarcoma arising at other sites, anatomical constraints in the retroperitoneum limit the ability to achieve wide resection margins. As a consequence, local recurrences in RPS are more frequent than in extremity sarcoma and comprise the leading cause of death especially for low-to-intermediate grade tumors, e.g., liposarcoma, the histopathological subtype of approximately one-half of sarcomas arising at this site. Conceptually, adjuvant radiation therapy (RT) is attractive, but this modality also is limited by anatomical constraints as well as the typically large size of these tumors at presentation. RT benefits in RPS remain to be conclusively demonstrated; its application is reserved for selected cases. In the report by McBride et al. ${ }^{1}$ an interesting approach is proposed, consisting of external preoperative therapy followed by intraoperative dose escalation with brachytherapy. This is an appealing concept, with the caveat that the justification for this strategy is tempered by the retrospective nature of their analysis and the small numbers of patients in their series. Moreover, patients presenting with recurrent tumor whose outcome is usually worse and less likely to be affected by any treatment modalities were nonetheless included in this study. With recurrence, the likelihood of definitive local control reduces to the anecdotal; therefore, the initial therapeutic intervention is critical if durable control (let alone cure) is

(C) Society of Surgical Oncology 2013

Published Online: 13 April 2013

A. Gronchi, MD

e-mail: alessandro.gronchi@istitutotumori.mi.it to result. McBride and colleagues used this opportunity to investigate possible predictors of survival, identifying multifocality as the only determinant of outcome. This finding is not surprising in that multifocality may be a reflection of an especially unfavorable underlying biology of RPS, with obvious prognostic impact, as we and others also have reported. ${ }^{2}$ Understanding the relative contributions of the effectiveness of local therapy against the inherent biology of specific RPS subtypes will be critical if we are to move a new set-point in RPS, in which specifically useful treatment options can be tailored to the individual patient.

\section{QUALITY OF LOCAL THERAPY}

\section{Surgical Strategy}

Some authors have advocated for the widest possible surgical resection at presentation, a strategy at variance with past recommendations advocating grossly complete extirpation in which the need or utility of resecting adjacent organs was limited to unequivocal direct tumor involvement. $^{3,4}$ These retrospective analyses have favored a more aggressive adjacent organ resection approach, reporting greater than $75 \%$ local control at 5 years. The need to standardize these seemingly disparate surgical strategies has been addressed recently by a panel of European and North American experts who have described how these tumors might be optimally approached. In essence, this surgical strategy consists of liberal en bloc resection of surrounding adjacent organs, even if not directly infiltrated, tempered by considerations of underlying histological subtype. ${ }^{5}$

The dilemma is a genuine concern about the potential morbidity of extended resections balanced against the 
expected survival benefits. Such extended surgery may indeed confer an increased potential for cure in low- and intermediate-grade tumors in which survival largely depends on local control. ${ }^{6}$ However, as intuitive as it may be, the final utility of this approach needs to be confirmed. In high-grade tumors, which generally have a more aggressive biology characterized by metastatic avidity, it can be anticipated that extended surgery alone will probably not significantly impact overall or disease-free survival. Manifestly, patients affected by any of these tumors are best treated by surgeons who are comfortable and familiar with the uncertainties of these biological issues and how such vagaries impact multidisciplinary approaches to these diseases.

\section{Radiation Therapy}

As correctly pointed out by McBride et al., ${ }^{7,8}$ given that RT has a demonstrated benefit in the treatment of soft tissue sarcoma of the extremity and trunk, there is strong reason to believe that it may similarly benefit those with RPS. Nevertheless, this has never been demonstrated in any prospective trial. Radiotherapy appears to improve local control as reported in several retrospective studies; 9,10 however, this potential advantage must be weighed against potential side effects. Most series favor the use of external beam RT in the preoperative setting, because it is well tolerated and can be administered at a total dose of 50.4 Gy. Postoperative RT usually is no longer considered given that effective doses (60 Gy or higher) cannot be delivered to the tumor bed, which is usually occupied or obstructed by radiosensitive normal organs (e.g., small bowel, kidney, liver) after surgery.

The above considerations undergirded the proposed treatment randomization of the American College of Surgeons Oncology Group (ACOSOG) Z9031 trial as advanced in the mid 1990s. If this trial had been allowed to accrue patients to the proposed end points, it might have been possible to determine whether or not the addition of preoperative radiotherapy in patients slated to receive surgical resection conferred survival benefits with acceptable thresholds of toxicity. Unfortunately, this trial was closed due to an unsatisfactory rate of patient accrual. The European Organization for Research and Treatment of Cancer (EORTC) has recently undertaken a similar trial, with the same basic design. Results are eagerly awaited and will hopefully provide more robust data to define an internationally accepted standard approach to this disease. ${ }^{11}$ While awaiting trial completion, patients might receive RT on an individualized basis especially in the preoperative setting, hopefully with prospective data retrieval on a registry basis. However,
RT cannot be utilized as a tool effective in limiting the extent of surgery.

\section{BIOLOGY OF THE TUMOR}

\section{Histological Subtype}

RPS is not a single disease entity. Even the commonest subtype, liposarcoma, cannot be considered as a single malignancy and is further subdivided into at least four distinctive tumor biologic categories: atypical lipomatous tumor, also referred to as well differentiated liposarcoma, dedifferentiated liposarcoma, myxoid/round cell liposarcoma, and pleomorphic liposarcoma. ${ }^{12}$ Taken together, these liposarcoma subtypes account for $50 \%$ of all RPS.

The approach to these different types of liposarcoma must be individualized, although this remains an area of unresolved controversy. Some authors advocate a more conservative approach when dealing with pure atypical lipomatous tumor given the very indolent biology of this disease. ${ }^{13,14}$ In contrast, most authors agree that extended surgery is the treatment of choice for dedifferentiated liposarcoma. More liberal use of RT as well as chemotherapeutic agents, such as anthracyclines and/or trabectedin, is usually proposed for the few patients affected by primary myxoid/ round cell retroperitoneal liposarcoma given the observed sensitivity of this tumor to both RT and chemotherapy in large, retrospective series. ${ }^{15-17}$

The second most common retroperitoneal histological subtype is leiomyosarcoma, typically arising from a major vessel, such as the inferior vena cava. Hematogenous spread, chiefly to the liver and/or the lungs, informs prognosis. Accordingly, systemic therapies should receive serious consideration, given this metastatic proclivity, although their efficacy remains to be demonstrated. Similarly, pleomorphic or undifferentiated sarcoma, malignant peripheral nerve sheath tumor, and synovial sarcoma all bear significant risks of dissemination; this reality should be factored into decisions regarding treatment approach. The trade-off between expected benefits versus morbidity must be carefully balanced when contemplating RT as a therapeutic adjunct in patients affected by high-grade RPS.

\section{Multifocality/Multicentricity}

Patterns of presentation, especially in retroperitoneal liposarcoma, may confer useful information about extent of resection. It is now well established that multifocal disease presentation, either in the de novo or recurrence contexts, is unfavorably associated with most survival parameters, including disease-specific and overall survival. ${ }^{2}$ What is yet to be determined (unpublished data; REP) is whether or 
not primary or recurrent multifocal disease presenting in several noncontiguous or even distinct, isolated, and/or remote intra-abdominal/retroperitoneal loci confers the same (or possibly worse) prognosis compared with multifocal primary or recurrent disease presenting in intraabdominal/retroperitoneal locations that are immediately adjacent to each other.

The biological implications of such differences may be particularly important for well-differentiated liposarcoma regarding the anticipated extent of resection. If multifocal, multicentric recurrence commonly occurs, it may represent a biological process in which any intra-abdominal/retroperitoneal fat-containing tissues are at risk for subsequent development of recurrence, whereas if rare then direct tumor extension processes may be at play. Under these latter circumstances, more aggressive local strategies, including extended resections involving contiguous organs suspicious for possibly harboring disease as well as RT, may be warranted. This unresolved controversy may be best addressed by a multicenter effort to record data prospectively for patterns of recurrence, taking care to differentiate multicentric, multifocal from multifocal failures confined to a single location.

At this time, complete surgical extirpation of RPS at the first operation remains the best therapy. Every attempt should be made to refer such patients to tertiary centers where the needed surgical oncology expertise resides. The disparate biological behaviors of the various histological subtypes must be accommodated in the overall therapeutic approach; not all RPS are the same. The EORTC RPS trial (see above) may definitively address the possible role of preoperative RT in this disease. Were RT proven to be effective in RPS patients, the combination of best surgery and best RT would hopefully yield improved results using treatment approaches already at our disposal. We can get to this point fastest if all major sarcoma institutions were able to make the effort to help accrue patients to this EORTC trial!

The world is shrinking rapidly, and we should vigorously embrace these and other opportunities to work together, ultimately for the benefit of sarcoma practitioners and patients everywhere.

\section{REFERENCES}

1. McBride SM, Raut CP, Lapidus M, Devlin PM, Marcus KJ, Bertagnolli $\mathrm{M}$, et al. Loco-regional recurrence after pre-operative radiation therapy for retroperitoneal sarcoma: adverse impact of multifocal disease and potential implications of dose escalation. Ann Surg Oncol. 2013. doi:10.1245/s10434-013-2868-y.
2. Anaya DA, Lahat G, Liu J, Xing Y, Cormier JN, Pisters PW, et al. Multifocality in retroperitoneal sarcoma: a prognostic factor critical to surgical decision-making. Ann Surg. 2009;249(1): $137-42$.

3. Gronchi A, Lo Vullo S, Fiore M, Mussi C, Stacchiotti S, Collini $\mathrm{P}$, et al. Aggressive surgical policies in a retrospectively reviewed single-institution case series of retroperitoneal soft tissue sarcoma patients. J Clin Oncol. 2009;27:24-30.

4. Bonvalot S, Rivoire M, Castaing M, Stoeckle E, Le Cesne A, Blay JY, et al. Primary retroperitoneal sarcomas: a multivariate analysis of surgical factors associated with local control. J Clin Oncol. 2009;27:31-37.

5. Bonvalot S, Raut CP, Pollock RE, Rutkowski P, Strauss DC, Hayes AJ, et al. Technical considerations in surgery for retroperitoneal sarcomas: position paper from E-surge, a master class in sarcoma surgery, and EORTC-STBSG. Ann Surg Oncol. 2012;19(9):2981-91.

6. Gronchi A, Miceli R, Colombo C, Stacchiotti S, Collini P, Mariani L, et al. Frontline extended surgery is associated with improved survival in retroperitoneal low-intermediate grade soft tissue sarcomas. Ann Oncol. 2012;23(4):1067-73.

7. Pisters PW, Harrison LB, Leung DH, Woodruff JM, Casper ES, Brennan MF. Long-term results of a prospective randomized trial of adjuvant brachytherapy in soft tissue sarcoma. J Clin Oncol. 1996;14(3):859-68.

8. Yang JC, Chang AE, Baker AR, Sindelar WF, Danforth DN, Topalian SL, et al. Randomized prospective study of the benefit of adjuvant radiation therapy in the treatment of soft tissue sarcomas of the extremity. J Clin Oncol. 1998;16(1):197-203.

9. Pawlik TM, Pisters PW, Mikula L, Feig BW, Hunt KK, Cormier $\mathrm{JN}$, et al. Long-term results of two prospective trials of preoperative external beam radiotherapy for localized intermediate- or high-grade retroperitoneal soft tissue sarcoma. Ann Surg Oncol. 2006;13(4):508-17.

10. Le Péchoux C, Musat E, Baey C, Al Mokhles H, Terrier P, Domont J, et al. Should adjuvant radiotherapy be administered in addition to front-line aggressive surgery (FAS) in patients with primary retroperitoneal sarcoma? Ann Oncol. 2012. doi:10.1093/ annonc/mds516.

11. http://clinicaltrials.gov/NCT01344018. Accessed 15 Feb 2013.

12. Dalal KM, Kattan MW, Antonescu CR, Brennan MF, Singer S. Subtype specific prognostic nomogram for patients with primary liposarcoma of the retroperitoneum, extremity, or trunk. Ann Surg. 2006;244(3):381-91.

13. Anaya DA, Lahat G, Wang X, Xiao L, Tuvin D, Pisters PW, et al. Establishing prognosis in retroperitoneal sarcoma: a new histology-based paradigm. Ann Surg Oncol. 2009;16(3):667-75.

14. Lahat G, Anaya DA, Wang X, Xiao L, Tuvin D, Pisters PW, et al. Resectable well-differentiated versus dedifferentiated liposarcomas: two different diseases possibly requiring different treatment approaches. Ann Surg Oncol. 2008;15(6):1585-93.

15. Jones RL, Fisher C, Al-Muderis O, Ashley S, Scurr M, Judson IR. Differential sensitivity of liposarcoma subtypes to chemotherapy. Eur J Cancer. 2005;41(18):2853-60.

16. Grosso F, Jones RL, Demetri GD, Judson IR, Blay JY, Le Cesne A, et al. Efficacy of trabectedin (ecteinascidin-743) in advanced pretreated myxoid liposarcomas: a retrospective study. Lancet Oncol. 2007;8(7):595-602.

17. Chung PW, Deheshi BM, Ferguson PC, Wunder JS, Griffin AM, Catton $\mathrm{CN}$, et al. Radiosensitivity translates into excellent local control in extremity myxoid liposarcoma: a comparison with other soft tissue sarcomas. Cancer. 2009;115(14):3254-61. 